\title{
A sufficient condition for nonrigidity of Carnot groups
}

\author{
Alessandro Ottazzi
}

Received: 30 August 2006 / Accepted: 16 July 2007 / Published online: 21 August 2007

C) Springer-Verlag 2007

\begin{abstract}
In this article we consider contact mappings on Carnot groups. Namely, we are interested in those mappings whose differential preserves the horizontal space, defined by the first stratum of the natural stratification of the Lie algebra of a Carnot group. We give a sufficient condition for a Carnot group $\mathrm{G}$ to admit an infinite dimensional space of contact mappings, that is, for $\mathrm{G}$ to be nonrigid. A generalization of Kirillov's Lemma is also given. Moreover, we construct a new example of nonrigid Carnot group.
\end{abstract}

Mathematics Subject Classification (2000) 22E25 - 22E60 · 53D10

\section{Introduction}

We are interested in the study of contact mappings on Carnot groups. In particular, we give a sufficient condition for a Carnot group to have an infinite dimensional space of contact mappings (Theorem 1). When this happens, the group is called nonrigid. The study of contact mappings has consequences in the theory of quasiconformal mappings and nonintegrable differential systems. For this reason contact mappings have been studied in several examples of Carnot groups. A remarkable piece of work concerning this circle of ideas is by Yamaguchi [10]. The main result leads to a complete classification of the rigid nilpotent Lie groups which arise as nilpotent parts of parabolic subgroups. In [5] Reimann showed that H-type groups are rigid provided that the dimension of the center is strictly greater than two. In a recent work, Cowling et al. [2] have proved rigidity results for homogeneous spaces G/P with $\mathrm{G}$ simple and $\mathrm{P}$ minimal parabolic. This is the situation considered by Yamaguchi, but their

This research was partly supported by the Swiss National Science Foundation. The author would like to thank H. M. Reimann for the helpful advices and the constant support.

A. Ottazzi

Mathematisches Institut, Universität Bern, Sidlerstr. 5, 3012 Bern, Switzerland

A. Ottazzi $(\varangle)$

DIMA, Università di Genova, Via Dodecaneso 35, 16146 Genova (1), Italy

e-mail: ottazzi@dima.unige.it 
results are independent of classification and rely on entirely elementary techniques. In [4], the author applied some of the techniques used in [2] to treat the case of Hessenberg manifolds, that can be locally viewed as quotients of Iwasawa nilpotent Lie groups and that turn out to be rigid in most cases. In [9] Warhurst in shows that jet spaces are nonrigid Carnot groups. His result is important because most of the examples of nonrigid groups that are known fall in this class.

Complete results for rigidity of Carnot groups do not seem to be within reach yet. In this paper we make a first attempt to give a result that holds for Carnot groups in general. Further (Sect. 3), we present an example of nonrigid Carnot group which is far from being a jet space. This example can be generalized to a class of nonrigid Hessenberg manifolds.

\section{Nonrigidity of Carnot groups: a sufficient condition}

Let $\mathfrak{g}$ be a Lie algebra over $\mathbb{R}$. The vector space $\mathfrak{z}:=\{X \in \mathfrak{g}:[X, \mathfrak{g}]=\{0\}\}$ is the center of $\mathfrak{g}$. A nilpotent Lie algebra $\mathfrak{g}$ has a $p$-step stratification if it can be written as

$$
\mathfrak{g}=\oplus_{i=1}^{p} \mathfrak{g}_{i},
$$

a direct sum of vector spaces such that

$$
\left[\mathfrak{g}_{1}, \mathfrak{g}_{j}\right]=\mathfrak{g}_{j+1},
$$

for every $j \geq 1$. An ideal $\mathfrak{I}$ in $\mathfrak{g}$ is stratified if $\mathfrak{I}=\oplus_{j=1}^{p} \mathfrak{I}_{j}$ with $\mathfrak{I}_{j}=\mathfrak{I} \cap \mathfrak{g}_{j}$ and $\left[\mathfrak{I}_{j}, \mathfrak{g}_{1}\right]=\mathfrak{I}_{j+1}$ for every $j=1, \ldots, p-1$. A Carnot group $\mathrm{G}$ is a connected, simply connected nilpotent Lie group, whose Lie algebra is stratified and equipped with an inner product such that $\mathfrak{g}_{i} \perp \mathfrak{g}_{j}, i \neq j$. By left translation, $\mathfrak{g}$ defines the tangent bundle TG to $\mathrm{G}$ and the subspace $\mathfrak{g}_{1}$ defines a subbundle HG of TG which is called horizontal bundle or contact bundle. Equation (1) implies that the horizontal bundle has the property that it generates at each point the whole tangent space to G. A diffeomorphism

$$
\phi: \mathcal{U} \rightarrow \mathcal{V}
$$

between open sets of $\mathrm{G}$ is called a contact mapping if the differential $\phi_{*}$ preserves the horizontal bundle. By composition (when $\mathcal{U}=\mathcal{V}$ ), the space of contact mappings is a group. If the group of contact mappings is finite dimensional, then we say that $\mathrm{G}$ is rigid, whereas $\mathrm{G}$ is nonrigid if the group of contact mappings is infinite dimensional. The contact condition of a map can be read at the Lie algebra level. A contact vector field $\mathrm{V}$ on an open set of $\mathrm{G}$ is a smooth vector field which generates a local one parameter flow $\phi_{t}$ of contact mappings. If $\tilde{X}$ is a left invariant vector field corresponding to a vector $X \in \mathfrak{g}_{1}$, we have

$$
\left.\frac{d}{d t}\left(\phi_{t}\right)_{*}(\tilde{X})\right|_{t=0}=-\mathcal{L}_{V}(\tilde{X})=[\tilde{X}, V],
$$

where $\mathcal{L}$ denotes the Lie derivative. Thus a smooth vector field $V$ is a contact vector field if and only if

$$
[V, \tilde{X}] \in \mathrm{HG}, \quad \text { for every } \tilde{X} \in \mathrm{HG},
$$

that is, ad $V$ preserves the horizontal bundle. Clearly, if a Carnot group $\mathrm{G}$ admits an infinite dimensional space of contact vector fields, then $\mathrm{G}$ is nonrigid. We use this observation for proving the following theorem.

Theorem 1 Let $G$ be a Carnot group with Lie algebra $\mathfrak{g}=\oplus_{i=1}^{p} \mathfrak{g}_{i}$. If there exists $X \in \mathfrak{g}_{1}$ such that $\operatorname{ad} X: \mathfrak{g} \rightarrow \mathfrak{g}$ has rank $\leq 1$, then $G$ is nonrigid. 
In order to prove the theorem, we need to state some properties of stratified Lie algebras. The following two lemmas are classical results, which hold for any nilpotent Lie algebra (see e.g. [1], Chap. 1).

Lemma 1 Let $\mathfrak{h}$ be a subalgebra of codimension 1 of a nilpotent Lie algebra $\mathfrak{g}$. Then $\mathfrak{h}$ is an ideal; in fact, $[\mathfrak{g}, \mathfrak{g}] \subset \mathfrak{h}$.

Lemma 2 (Kirillov's Lemma) Let $\mathfrak{g}$ be a noncommutative nilpotent Lie algebra whose center $\mathfrak{z}(\mathfrak{g})$ is one-dimensional. Then $\mathfrak{g}$ can be written as

$$
\mathfrak{g}=\mathbb{R} Z \oplus \mathbb{R} X \oplus \mathbb{R} Y \oplus \mathfrak{w}=\mathbb{R} Y \oplus \mathfrak{g}_{0},
$$

a vector space direct sum, where

$$
\mathbb{R} Z=\mathfrak{z}(\mathfrak{g}), \text { and }[X, Y]=Z ;
$$

and $\mathfrak{g}_{0}=\mathbb{R} X+\mathbb{R} Z+\mathfrak{w}$ is the centralizer of $X$, and an ideal.

Kirillov's Lemma points out that any nilpotent Lie algebra with one-dimensional center has a subalgebra which is a Heisenberg algebra. In the case in which $\mathfrak{g}$ is stratified with center of any dimension, we prove under a further assumption that $\mathfrak{g}$ has a subalgebra which is filiform, in a sense that we now specify.

Lemma 3 Let $\mathfrak{g}=\oplus_{i=1}^{p} \mathfrak{g}_{i}$ be a stratified nilpotent Lie algebra. Suppose that there exists $X \in \mathfrak{g}_{1}$ such that $\operatorname{rank}(\operatorname{ad} X)=1$. Then $\mathfrak{g}$ can be written as

$$
\mathfrak{g}=\mathbb{R} Z_{1} \oplus \cdots \oplus \mathbb{R} Z_{m} \oplus \mathbb{R} X \oplus \mathbb{R} Y \oplus \mathfrak{w},
$$

a vector space direct sum, where

$$
\begin{aligned}
{[X, Y] } & =Z_{1}, \\
{\left[Z_{i}, Y\right] } & =Z_{i+1}, i=1, \ldots, m-1, \\
Z_{m} & \in \mathfrak{z}(\mathfrak{g}) .
\end{aligned}
$$

Moreover,

$$
\mathfrak{g}_{0}=\mathbb{R} Z_{1} \oplus \cdots \oplus \mathbb{R} Z_{m} \oplus \mathbb{R} X \oplus \mathfrak{w}
$$

is an ideal and

$$
\Im=\mathbb{R} Z_{1} \oplus \cdots \oplus \mathbb{R} Z_{m} \oplus \mathbb{R} X
$$

is abelian. Finally, $\mathfrak{g}_{1}$ has a basis $\left\{X, Y, U_{1}, \ldots, U_{s}\right\}$ so that

$$
\begin{aligned}
{\left[X, U_{i}\right] } & =0, \quad \forall i=1, \ldots, s \\
{\left[Z_{j}, U_{i}\right] } & =0, \quad \forall j=1, \ldots, m, \quad \forall i=1, \ldots, s .
\end{aligned}
$$

Proof Since $\operatorname{rank}(\operatorname{ad} X)=1$, it follows that $[X, \mathfrak{g}]=\mathbb{R} Z_{1}$ for some $Z_{1} \in \mathfrak{g}$. Since $\mathfrak{g}_{1}$ generates $\mathfrak{g}$, it follows that there exists $Y \in \mathfrak{g}_{1}$ such that $0 \neq[X, Y] \in \mathbb{R} Z_{1}$, for otherwise $X$ would be in the center of $\mathfrak{g}$. Let $\alpha: \mathfrak{g} \rightarrow \mathbb{R}$ be the linear map defined by $[X, W]=\alpha(W) Z_{1}$ and choose $Y$ so that $\alpha(Y)=1$. Let $\mathfrak{g}_{0}=\operatorname{ker} \alpha$ and notice that $\operatorname{ker} \alpha=\operatorname{ker}$ ad $X$. Define $Z_{i}=\left[Z_{i-1}, Y\right]$ for every $i=2, \ldots, m$, where $m$ is the lowest index for which $\left[Z_{m}, Y\right]=0$. Clearly $X \in \mathfrak{g}_{0}$. Moreover, $Z_{1}, \ldots, Z_{m}$ are in $\mathfrak{g}_{0}$. Indeed, if $(\operatorname{ad} X) Z_{1} \neq 0$, then $\left[X, Z_{1}\right]=$ $\lambda Z_{1}$ because $[X, \mathfrak{g}]=\mathbb{R} Z_{1}$. This implies that $(\operatorname{ad} X)^{k} Z_{1}=\lambda^{k} Z_{1}$ for every positive integer $k$, whence ad $X$ is not nilpotent, a contradiction. Therefore $Z_{1} \in \mathfrak{g}_{0}$ and using induction and 
Jacobi's identity it is easy to see that $\left[X, Z_{j}\right]=0$ for every $j \in\{2, \ldots, m\}$. The vector $X$ and $Z_{1}, \ldots, Z_{m}$ are linearly independent because they all belong to different strata of $\mathfrak{g}$. Let $\mathfrak{w}$ be a complementary subspace to $\mathbb{R} Z_{1} \oplus \cdots \oplus \mathbb{R} Z_{m} \oplus \mathbb{R} X$ in $\mathfrak{g}_{0}$. The space $\mathfrak{g}_{0}$ is clearly a subalgebra. Then Lemma 1 implies that $\mathfrak{g}_{0}$ is an ideal.

Take now $U_{1}, \ldots, U_{s} \in \mathfrak{g}_{1} \cap \mathfrak{g}_{0}$ such that $\left\{X, Y, U_{1}, \ldots, U_{s}\right\}$ is a basis of $\mathfrak{g}_{1}$. It follows that $\left[X, U_{i}\right]=0, \forall i \in\{1, \ldots, s\}$. In order to show that $\left[Z_{j}, U_{i}\right]=0$ for every $i \in\{1, \ldots, s\}$ and every $j \in\{1, \ldots, m\}$, we first use induction to prove that

$$
\left[(\operatorname{ad} Y)^{k} X, W\right]=0, \quad \forall k \geq 0, \forall W \in \mathfrak{g}_{0} .
$$

If $k=0$, then (8) is obvious. Suppose now that $\left[(\operatorname{ad} Y)^{k} X, W\right]=0$ for every $W \in \mathfrak{g}_{0}$. Then

$$
\begin{aligned}
{\left[(\operatorname{ad} Y)^{k+1} X, W\right] } & =\left[(\operatorname{ad} Y)(\operatorname{ad} Y)^{k} X, W\right] \\
& =\left[Y,\left[(\operatorname{ad} Y)^{k} X, W\right]\right]-\left[(\operatorname{ad} Y)^{k} X,[Y, W]\right]=0,
\end{aligned}
$$

because $[Y, W] \in \mathfrak{g}_{0}$. Since

$$
\begin{aligned}
{\left[Z_{k}, U_{i}\right] } & =\left[\left[Z_{k-1}, Y\right], U_{i}\right] \\
& =-\left[\left[Z_{k-1}, U_{i}\right], Y\right]-\left[\left[U_{i}, Y\right], Z_{k-1}\right] \\
& =0-\left[\left[U_{i}, Y\right],\left[Z_{k-2}, Y\right]\right] \\
& =-\left[\left[U_{i}, Y\right],[[\ldots,[[X, Y], Y], \ldots, Y]]\right],
\end{aligned}
$$

it follows from (8) that $\left[Z_{k}, U_{i}\right]=0$ for every $i \in\{1, \ldots, s\}$ and every $k \in\{1, \ldots, m\}$. In particular, since $Z_{m}$ commutes with $Y$ by construction, we have that $Z_{m}$ commutes with $\mathfrak{g}_{1}$ and therefore it is in the center of $\mathfrak{g}$. We conclude the proof observing that $\mathfrak{I}$ is abelian. In fact, $X$ commutes with $Z_{j}, j=1, \ldots, m$. Moreover, $\left[Z_{i}, Z_{j}\right]=0, i, j=1, \ldots, m$ because of (8).

Given a vector $W$ in $\mathfrak{g}$, the corresponding left invariant vector field is given by

$$
(\tilde{W} f)(g)=\left.\frac{d}{d t}\right|_{t=0} f(g \cdot \exp t W),
$$

where $g \in \mathrm{G}$ and $f$ is a smooth function on $\mathrm{G}$. We put on $\mathrm{G}$ exponential coordinates

$$
g=\left(w_{1}, \ldots, w_{n}\right)=\exp \left(w_{1} W_{1}+\cdots+w_{n} W_{n}\right),
$$

where $\left\{W_{1}, \ldots, W_{n}\right\}$ is a basis of $\mathfrak{g}$. When working with stratified Lie algebras, it is usually convenient to choose a basis that respects the stratification, that is a collection of bases of each stratum $\mathfrak{g}_{i}$. Once we choose such a basis and we take a basis vector $W \in \mathfrak{g}_{1}$, a straightforward calculation gives

$$
\tilde{W}=\frac{\partial}{\partial w}+D
$$

where $w$ denotes the coordinate of a point of $\mathfrak{g}$ along $W$ and $D$ is a first order differential operator containing derivatives along coordinates corresponding to vectors that lie on strata strictly higher than one (for a detailed description of a left invariant vector field basis of a Carnot group see e.g. [7]). Now we can prove Theorem 1.

Proof If $\operatorname{rank}(\operatorname{ad} X)=0$, then $X \in \mathfrak{z}(\mathfrak{g})$. Therefore, $V=f X$ is a contact vector field for every smooth function $f$, because (2) is easily verified.

Set now $\operatorname{rank}(\operatorname{ad} X)=1$. Define $Y, U_{1}, \ldots, U_{s} \in \mathfrak{g}_{1}$ and $Z_{1}, \ldots, Z_{m}$ as in Lemma 3. Further, let $\tilde{X}, \tilde{Y}, \tilde{U}_{1}, \ldots, \tilde{U}_{s}, \tilde{Z}_{1}, \ldots, \tilde{Z}_{m}$ be the corresponding left invariant vector fields. 
In the following, we will write these vector fields by dropping the $\sim$ and thus we identify the Lie algebra elements with the left invariant vector fields. Similarly, we write $\mathfrak{g}_{1}$ for HG.

Consider the vector field on $\mathrm{G}$ defined as

$$
V=Y^{m} f X+Y^{m-1} f Z_{1}+\cdots+Y f Z_{m-1}+f Z_{m},
$$

We show that $V$ is a contact vector field for every smooth function $f=f(y)$, concluding that $\mathrm{G}$ is nonrigid. To this end, we study the brackets of $V$ with the left invariant vector fields corresponding to the chosen basis of $\mathfrak{g}_{1}$. First,

$$
[V, X]=\left[Y^{m} f X+Y^{m-1} f Z_{1}+\cdots+Y f Z_{m-1}+f Z_{m}, X\right]=0,
$$

because $X$ commutes with $Z_{1}, \ldots, Z_{m}$ and from (9) it annihilates the coefficients of $V$. Further, by (3), (4) and (5) we have

$$
\begin{aligned}
{[V, Y]=} & {\left[Y^{m} f X+Y^{m-1} f Z_{1}+\cdots+Y f Z_{m-1}+f Z_{m}, Y\right] } \\
= & Y^{m} f Z_{1}+Y^{m-1} f Z_{2}+\cdots+Y f Z_{m}-Y^{m+1} f X \\
& -Y^{m} f Z_{1}-Y^{m-1} f Z_{2}-\cdots-Y f Z_{m} \\
= & -Y^{m+1} f X,
\end{aligned}
$$

which implies $[V, Y] \in \mathfrak{g}_{1}$. Finally, from (6), (7) and (9) we have

$$
\left[V, U_{i}\right]=\left[Y^{m} f X+Y^{m-1} f Z_{1}+\cdots+Y f Z_{m-1}+f Z_{m}, U_{i}\right]=0,
$$

for every $i \in\{1, \ldots, s\}$.

The examples of nonrigid Carnot groups in the literature are jet spaces and the complexified Heisenberg group. We show that their nonrigidity follows from Theorem 1. Also, we observe that quotients of jet spaces by normal stratified subgroups are nonrigid.

Example 1 If $\mathfrak{g}$ is a complex Lie algebra, then Lemmas 1, 2 and 3 still hold. The following restatement of Theorem 1 is true, with the same proof.

Corollary 1 Let $\mathfrak{g}$ be a complex stratified nilpotent Lie algebra. Suppose that there exists $X \in \mathfrak{g}_{1}$ such that $\operatorname{ad} X$ has rank $\leq 1$, viewed as a homomorphism between complex vector spaces. Then there exists an infinite dimensional family of complex vector fields $V$ satisfying (2).

A consequence of Corollary 1 is that the complexified Heisenberg group, viewed as a real Carnot group, is nonrigid. This result was proved in [6]. We show here that nonrigidity follows from the rank one condition given in the corollary above. Let $\left\{W_{1}, W_{2}, Z\right\}$ be an orthonormal basis of the Heisenberg algebra, with $\left[W_{1}, W_{2}\right]=Z$. The complexified Lie algebra $\mathfrak{g}_{\mathbb{C}}$ is obtained by taking these three vectors to be the generators of a complex Lie algebra with the same bracket relations. The complexified Heisenberg group is the connected complex Lie group $\mathbb{G}_{\mathbb{C}}$ with Lie algebra $\mathfrak{g} \mathbb{C}$. As a model space for $\mathfrak{G}_{\mathbb{C}}$ we use $\mathbb{C}^{3}=\left\{\left(w_{1}, w_{2}, z\right)\right\}$ with the multiplication law derived from Baker-Campbell-Hausdorff formula. The left invariant vector fields corresponding to the chosen basis are:

$$
\begin{aligned}
W_{1} & =\frac{\partial}{\partial w_{1}}-\frac{1}{2} w_{2} \frac{\partial}{\partial z} \\
W_{2} & =\frac{\partial}{\partial w_{2}}+\frac{1}{2} w_{1} \frac{\partial}{\partial z} \\
Z & =\frac{\partial}{\partial z} .
\end{aligned}
$$


Then ad $W_{1}$ has complex rank one and from the proof of Theorem 1 we have that $V=f Z+$ $W_{2} f W_{1}$ satisfies (2) for every complex valued smooth function $f=f\left(w_{2}\right)$. In particular, let $f$ be a holomorphic function of $w_{2}$. We now look at $\mathfrak{g}_{\mathbb{C}}$ as a real Lie algebra $\mathfrak{g}$ (with corresponding group $\mathrm{G}$ ) with complex structure $J$ derived from multiplication by $i$. This algebra is then generated as a vector space by

$$
X_{1}, X_{2}, X_{3}=J X_{1}, X_{4}=J X_{2}, Z_{1}, Z_{2}=J Z_{1}
$$

where $w_{1}=x_{1}+i x_{3}, w_{2}=x_{2}+i x_{4}, z=z_{1}+i z_{2}$ and therefore

$$
X_{1}=2 \operatorname{Re} W_{1}, \quad X_{3}=-2 \operatorname{Im} W_{1}, \quad X_{2}=2 \operatorname{Re} W_{2}, \quad X_{4}=-2 \operatorname{Im} W_{2} .
$$

The nontrivial bracket relations are

$$
\begin{aligned}
& Z_{1}=\left[X_{1}, X_{2}\right]=-\left[X_{3}, X_{4}\right] \\
& Z_{2}=\left[X_{1}, X_{4}\right]=-\left[X_{2}, X_{3}\right] .
\end{aligned}
$$

The bracket is $J$-invariant:

$$
[J X, Y]=J[X, Y],
$$

for every $X, Y \in \mathfrak{g}$. So $\mathfrak{g}=\mathfrak{h} \oplus \mathfrak{z}$, with $\mathfrak{h}=\operatorname{span}\left\{X_{1}, X_{2}, X_{3}, X_{4}\right\}$ and $\mathfrak{z}=\operatorname{span}\left\{Z_{1}, Z_{2}\right\}$ so that one has $[\mathfrak{h}, \mathfrak{h}]=\mathfrak{z}$ and $[\mathfrak{h}, \mathfrak{z}]=[\mathfrak{z}, \mathfrak{z}]=0$. Then, a vector field $F$ on $\mathrm{G}$ is a contact vector field if it satisfies

$$
\left[F, X_{i}\right] \in \operatorname{span}\left\{X_{1}, X_{2}, X_{3}, X_{4}\right\},
$$

for every $i=1, \ldots, 4$. We prove that the real and imaginary parts of $V$ are contact vector fields for every holomorphic function $f$ of $w_{2}$, from which it follows that $\mathrm{G}$ is nonrigid. Writing $f\left(w_{2}\right)=f\left(x_{2}, x_{4}\right)=u\left(x_{2}, x_{4}\right)+i v\left(x_{2}, x_{4}\right)$, a direct calculation gives

$$
V_{1}:=\operatorname{Re} V=u Z_{1}+v Z_{2}+X_{2} u X_{1}-X_{4} u X_{3}
$$

and

$$
V_{2}:=\operatorname{Im} V=v Z_{1}-u Z_{2}-X_{4} u X_{1}-X_{2} u X_{3}
$$

where we used $X_{2} u=X_{4} v$ and $X_{4} u=-X_{2} v$, which follow from Cauchy-Riemann equations for $f$. It is now straightforward to verify that $V_{1}$ and $V_{2}$ satisfy (11).

Example 2 In [9] Warhurst showed that jet spaces are nonrigid Carnot groups. Theorem 1 provides a different proof for nonrigidity of jet spaces. Some classical groups that are nonrigid can be viewed as jet spaces, such as the Heisenberg group and the Engel group.

We recall the definition and some standard facts about jet spaces. For more details, see [8]. A function $f: \mathbb{R}^{m} \rightarrow \mathbb{R}$ has $d(m, k)=\left(\begin{array}{c}m+k-1 \\ k\end{array}\right)$ distinct $k$ th order partial derivatives

$$
\partial_{I} f(p)=\frac{\partial^{k} f}{\partial x_{1}^{i_{1}} \cdots \partial x_{m}^{i_{m}}}(p)
$$

where $I=\left(i_{1}, \ldots, i_{m}\right)$ satisfies $|I|=i_{1}+\cdots+i_{m}=k$. We denote the set of $k$-indexes by $I(k)$ and write $\tilde{I}(k)=I(0) \cup \cdots \cup I(k)$. For $I \in \tilde{I}(k)$ and $t \in \mathbb{R}^{m}$ we define

$$
I !=i_{1} ! i_{2} ! \cdots i_{m} ! \text { and } t^{I}=\left(t_{1}\right)^{i_{1}}\left(t_{2}\right)^{i_{2}} \ldots\left(t_{m}\right)^{i_{m}} .
$$

Moreover, the $k$ th order Taylor polynomial of $f$ at $p$ is given by

$$
T_{p}^{k}(f)(t)=\sum_{I \in \tilde{I}(k)} \partial_{I} f(p) \frac{(t-p)^{I}}{I !} .
$$


If $D \subset \mathbb{R}^{m}$ is open and $p \in D$, then two functions $f_{1}, f_{2} \in C^{k}(D, \mathbb{R})$ are defined to be equivalent at $p$, denoted $f_{1} \sim_{p} f_{2}$, if and only if $T_{p}^{k}\left(f_{1}\right)=T_{p}^{k}\left(f_{2}\right)$. The $k$-jet space over $D$ is given by

$$
J^{k}(D, \mathbb{R})=\cup_{p \in D} C^{k}(D, \mathbb{R}) / \sim_{p}
$$

where elements are denoted $j_{p}^{k}(f)$. It comes equipped with the following projections

$$
x: J^{k}(D, \mathbb{R}) \rightarrow D \text { and } \pi_{j}^{k}: J^{k}(D, \mathbb{R}) \rightarrow J^{k-j}(D, \mathbb{R}), \quad j=1, \ldots, k,
$$

where

$$
x\left(j_{p}^{k}(f)\right)=p \quad \text { and } \quad \pi_{j}^{k}\left(j_{p}^{k}(f)\right)=j_{p}^{k-j}(f) .
$$

Global coordinates are given by $\psi^{(k)}=\left(x, u^{(k)}\right)$, where

$$
x\left(j_{p}^{k}(f)\right)=p, \quad u_{I}\left(j_{p}^{k}(f)\right)=\partial_{I} f(p), \quad I \in \tilde{I}(k),
$$

and

$$
u^{(k)}=\left\{u_{I}: I \in \tilde{I}(k)\right\} .
$$

It follows that

$$
J^{k}(D, \mathbb{R}) \equiv D \times \mathbb{R}^{d(m, 0)} \times \mathbb{R}^{d(m, 1)} \times \cdots \times \mathbb{R}^{d(m, k)} .
$$

If $f=\left(f^{1}, \ldots, f^{n}\right)$ is a map $f: D \rightarrow \mathbb{R}^{n}$ then we apply the jet apparatus to the coordinate functions $f^{l}$, and we end up with the trivialization

$$
J^{k}\left(D, \mathbb{R}^{n}\right) \equiv D \times \mathbb{R}^{n d(m, 0)} \times \mathbb{R}^{n d(m, 1)} \times \cdots \times \mathbb{R}^{n d(m, k)} .
$$

Global coordinates are again denoted by $\psi^{(k)}=\left(x, u^{(k)}\right)$, where

$$
x\left(j_{p}^{k}(f)\right)=p, \quad u_{I}^{l}\left(j_{p}^{k}(f)\right)=\partial_{I} f^{l}(p), \quad I \in \tilde{I}(k), l=1, \ldots, n,
$$

and

$$
u^{(k)}=\left\{u_{I}^{l}: I \in \tilde{I}(k), l=1, \ldots, n\right\} .
$$

At the tangent space level, one has a natural contact structure. Namely, the tangent space of $J^{k}\left(\mathbb{R}^{m}, \mathbb{R}^{n}\right)$ at the identity, denoted by $\mathfrak{X}$, is a stratified nilpotent Lie algebra which endows the jet space with the structure of a Carnot group. A stratified basis for $\mathfrak{X}$ is

$$
\begin{aligned}
& L_{0}=\operatorname{span}\left\{X_{j}: j=1, \ldots, m\right\} \oplus \operatorname{span}\left\{\frac{\partial}{\partial u_{I}^{l}}: I \in I(k), l=1, \ldots, n\right\}, \\
& L_{j}=\operatorname{span}\left\{\frac{\partial}{\partial u_{I}^{l}}: I \in I(k-j), l=1, \ldots, n\right\}, \quad \text { for every } j=1, \ldots, k,
\end{aligned}
$$

where $X_{j}=\frac{\partial}{\partial x_{j}}+\sum_{l=1}^{n} \sum_{I \in \tilde{I}(k-1)} u_{I+e_{j}}^{l} \frac{\partial}{\partial u_{I}^{l}}, j=1, \ldots, m$ and where $\left\{e_{j}\right\}_{j=1}^{m}$ is the canonical basis of $\mathbb{R}^{m}$. The non trivial commutators with respect to this basis are

$$
\left[\frac{\partial}{\partial u_{I+e_{j}}^{l}}, X_{j}\right]=\frac{\partial}{\partial u_{I}^{l}}, \quad I \in I(0) \cup \cdots \cup I(k-1),
$$

for every $j=1, \ldots, m$. From the bracket relations we see that $L_{j}=\left[L_{0}, L_{j-1}\right]$. 
Corollary 2 (i) The jet spaces $J^{k}\left(\mathbb{R}^{m}, \mathbb{R}^{n}\right)$ are nonrigid Carnot groups.

(ii) Let $\mathfrak{X}$ be the stratified Lie algebra of a jet space and $\mathfrak{I}$ a stratified ideal of $\mathfrak{X}$. Then the quotient $\mathfrak{X} / \mathfrak{I}$ is the Lie algebra of a nonrigid Carnot group.

Proof (i) By Theorem 1, it is enough to exhibit a vector $W$ in the first stratum of the stratification of the tangent space of $J^{k}\left(\mathbb{R}^{m}, \mathbb{R}^{n}\right)$ whose image under the adjoint representation has dimension one. Consider the vector $W=\frac{\partial}{\partial u_{I}^{l}}, I \in I(k)$, for some $l \in\{1, \ldots, n\}$. We set $I$ to have equal zero all components except one, which is then forced to be equal $k$. Let $\bar{j}$ be this nonzero component of $I$. Looking at (13), $W \in L_{0}$ commutes with every element in $L_{0}$ except $X_{\bar{J}}$. Thus ad $W$ has rank one and lies in $\mathfrak{g}_{1}$.

(ii) For $s=0, \ldots, k$, let us denote

$$
\begin{aligned}
A_{s}:= & \left\{\frac{\partial}{\partial u_{I}^{l}}: l=1, \ldots, n, \quad I=\left(i_{1}, \ldots, i_{m}\right) \in I(k)\right. \\
& \text { such that } \left.i_{\bar{J}}=k-s, \quad \text { for some } \bar{j} \in\{1, \ldots, m\}\right\} .
\end{aligned}
$$

Let $\pi: \mathfrak{X} \rightarrow \mathfrak{X} / \mathfrak{I}$ be the natural projection. Suppose the following property holds:

$$
\text { if } \begin{aligned}
\pi(W) & =0, \quad \forall W \in A_{s} ; \quad \forall s=0, \ldots, r ; r \leq k-1 \\
& \Longrightarrow \operatorname{rank}\left(\operatorname{ad} \pi\left(W^{\prime}\right)\right)=1, \quad \forall W^{\prime} \in A_{r+1}, \quad \text { with } \pi\left(W^{\prime}\right) \neq 0 .
\end{aligned}
$$

Set now $W \in A_{0}$. Then ad $W$ has rank one, so that $\operatorname{ad} \pi(W)$ has rank one too, unless $\pi(W)=0$. Therefore, $\mathfrak{X} / \mathfrak{I}$ is nonrigid unless $\pi(W)=0$ for every $W \in A_{0}$. Assume this. Using (14), we iterate the same argument in order to conclude that $\mathfrak{X} / \mathfrak{I}$ is nonrigid, unless $\pi\left(\frac{\partial}{\partial u_{I}^{l}}\right)=0$ for every $I \in I(k)$. Nevertheless, in this case the quotient $\mathfrak{X} / \mathfrak{I}$ would be the abelian Lie algebra generated as a vector space by the set of vectors $\left\{\pi\left(X_{j}\right): j=1, \ldots, m\right\}$ and therefore trivially nonrigid. Hence, the proof is finished provided we show (14). Set then $W^{\prime} \in A_{r+1}$. By (13) $\left[W^{\prime}, X_{j}\right]$ is a nonzero vector of $L_{1}$ if and only if $W^{\prime}=\frac{\partial}{\partial u_{I}^{l}}$ with $i_{j} \neq 0$ and $l \in\{1, \ldots, n\}$. In this case, $\left[W^{\prime}, X_{j}\right]=\frac{\partial}{\partial u_{I-e_{j}}^{l}}$. Let $\bar{j} \in\{1, \ldots, m\}$ such that $i_{\bar{j}}=k-(r+1)$, which exists by definition of $A_{r+1}$. Now, either $j=\bar{\jmath}$ or $j \neq \bar{\jmath}$. In the latter case, $\frac{\partial}{\partial u_{I-e_{j}}^{l}} \in \mathfrak{I}$, because there exists $W \in A_{r}$ such that

$$
\pi\left(\frac{\partial}{\partial u_{I-e_{j}}^{l}}\right)=\pi\left(\left[W, X_{\bar{J}}\right]\right)=\left[\pi(W), \pi\left(X_{\bar{J}}\right)\right]
$$

and $\pi(W)=0$ by hypothesis. Therefore, $\left[\pi\left(W^{\prime}\right), \pi\left(X_{j}\right)\right] \neq 0$ if and only if $j=\bar{\jmath}$. It follows that $\operatorname{ad} \pi\left(W^{\prime}\right)$ has rank one, as required.

Remark 1 Since Theorem 1 includes all nonrigid Carnot groups known so far, we may conjecture that the rank one condition is also a necessary condition for nonrigidity.

Using Theorem 1, it is possible to construct several examples of nonrigid Carnot groups. In the next section we give an example of nonrigid Carnot group, which is neither isomorphic to any jet space, nor to any quotient of a jet space. 


\section{A new example of nonrigid group}

Let $\mathfrak{g}=\mathbb{R}^{4} \oplus \mathbb{R}^{3}=\mathfrak{h} \oplus \mathfrak{z}$ be a step two stratified nilpotent Lie algebra defined by the following non zero brackets:

$$
\left[H_{1}, K_{1}\right]=Z_{1} \quad\left[K_{1}, K_{2}\right]=Z_{2} \quad\left[K_{2}, H_{2}\right]=Z_{3},
$$

where $\left\{H_{1}, H_{2}, K_{1}, K_{2}, Z_{1}, Z_{2}, Z_{3}\right\}$ is a basis of $\mathfrak{g}$. Let $\mathrm{G}$ be the connected and simply connected nilpotent Lie group whose Lie algebra is $\mathfrak{g}$. It is easy to check that ad $H_{1}$ has rank one, so that $\mathrm{G}$ is nonrigid by Theorem 1 .

\section{$3.1 \mathfrak{g}$ is not isomorphic to the Lie algebra of any jet space}

We show that $\mathfrak{g}$ is not isomorphic to the Lie algebra of any jet space. Since $\mathfrak{g}$ is a step two nilpotent Lie algebra, the only jet spaces possibly isomorphic to $\mathrm{G}$ are of the form $J^{1}\left(\mathbb{R}^{m}, \mathbb{R}^{n}\right)$. The Lie algebra of this space is

$$
\mathfrak{X}=L_{0} \oplus L_{1} .
$$

We simplify the notations for the basis of a jet space defined in (12) writing

$$
\begin{aligned}
& L_{0}=\operatorname{span}\left\{e_{1}, \ldots, e_{m}\right\} \oplus \operatorname{span}\left\{e_{1}^{1}, \ldots, e_{m}^{1}, \ldots, e_{1}^{n}, \ldots, e_{m}^{n}\right\}, \\
& L_{1}=\operatorname{span}\left\{e^{1}, \ldots, e^{n}\right\},
\end{aligned}
$$

where the nontrivial bracket relations are

$$
\left[e_{j}^{l}, e_{j}\right]=e^{l}
$$

for every $j=1, \ldots, m$ and $l=1, \ldots, n$. We now argue by contradiction, assuming that there exists a-strata preserving—Lie algebra isomorphism $\iota: \mathfrak{g} \rightarrow \mathfrak{X}$ for some choice of $m$ and $n$. Since $\iota$ preserves the stratification, $\operatorname{dim}(\mathfrak{z})=\operatorname{dim} L_{1}$, whence $n=3$. Moreover, since $\operatorname{dim}(\mathfrak{h})=\operatorname{dim} L_{0}$ we also have $\operatorname{dim}(\mathfrak{h})=4=m+n m=4 m$, implying $m=1$. Hence, the only possible jet space is $J^{1}\left(\mathbb{R}, \mathbb{R}^{3}\right)$, with

$$
L_{0}=\operatorname{span}\left\{e_{1}\right\} \oplus \operatorname{span}\left\{e_{1}^{1}, e_{1}^{2}, e_{1}^{3}\right\}, \quad L_{1}=\operatorname{span}\left\{e^{1}, e^{2}, e^{3}\right\},
$$

and

$$
\left[e_{1}^{1}, e_{1}\right]=e^{1}, \quad\left[e_{1}^{2}, e_{1}\right]=e^{2}, \quad\left[e_{1}^{3}, e_{1}\right]=e^{3} .
$$

Let $\mathfrak{g}^{*}$ and $\mathfrak{X}^{*}$ be the dual vector spaces of $\mathfrak{g}$ and $\mathfrak{X}$, respectively, and fix the bases dual to the given ones. Every $l \in \mathfrak{g}^{*}$ defines a natural bilinear form

$$
B_{l}: \mathfrak{g} \times \mathfrak{g} \rightarrow \mathbb{R}
$$

where $B_{l}(X, Y)=l([X, Y])$. The radical of $l$ is defined as

$$
\mathfrak{r}_{l}:=\left\{X \in \mathfrak{g}: B_{l}(X, Y)=0, \quad \forall Y \in \mathfrak{g}\right\} .
$$

Then $\iota\left(\mathfrak{r}_{l}\right)$ is the radical of the bilinear form associated to $\mathfrak{L}:=\left(\iota^{*}\right)^{-1} l \in \mathfrak{X}^{*}$, as the following chain of equalities shows, together with the fact that $\iota$ is an isomorphism:

$$
\left(\iota^{*}\right)^{-1} l\left([i(X), i(Y)]=\left(\iota^{*}\right)^{-1} l(i[X, Y])=l([X, Y])=0,\right.
$$

for every $X \in \mathfrak{r}_{l}$ and $Y \in \mathfrak{g}$. Set now $l=Z_{1}^{*}$ and $\mathfrak{L}=\left(\iota^{*}\right)^{-1}\left(Z_{1}^{*}\right)$. It is straightforward to verify that $\mathfrak{r}_{l}=\operatorname{span}\left\{K_{2}, H_{2}, Z_{1}, Z_{2}, Z_{3}\right\}$. Since

$$
\left[\iota\left(\mathfrak{r}_{l}\right), \iota\left(\mathfrak{r}_{l}\right)\right]=\iota\left[\mathfrak{r}_{l}, \mathfrak{r}_{l}\right]=\iota\left(\mathbb{R} Z_{3}\right),
$$


it follows that $\iota\left(\mathfrak{r}_{l}\right)$ is not commutative. This is possible only if it contains some element $\bar{e}$ with nonzero component along $e_{1}$. Therefore,

$$
\left(\iota^{*}\right)^{-1} l\left[\bar{e}, e^{\prime}\right]=\mathfrak{L}\left[\bar{e}, e^{\prime}\right]=0
$$

for every $e^{\prime} \in \mathfrak{X}$. But $[\bar{e}, \mathfrak{X}]=L_{1}$, whence $\mathfrak{L}\left(L_{1}\right)=0$. Now,

$$
\begin{aligned}
\mathfrak{L} & =\left(\iota^{*}\right)^{-1} l=\left(\iota^{*}\right)^{-1} Z_{1}^{*} \\
& \Longleftrightarrow \iota^{*}(\mathfrak{L})=Z_{1}^{*} \\
& \Longrightarrow 1=Z_{1}^{*}\left(Z_{1}\right)=\mathfrak{L}\left(\iota\left(Z_{1}\right)\right) .
\end{aligned}
$$

Since $\iota$ preserves the center, $\iota\left(Z_{1}\right)$ is in the center of $\mathfrak{X}$, which is $L_{1}$. Then the last arrow of the chain above gives a contradiction, because $\mathfrak{L}$ should be zero on $L_{1}$.

\subsection{Quotients of jet spaces}

We show that $\mathfrak{g}=\mathfrak{h} \oplus \mathfrak{z}$ is not isomorphic to the quotient of the Lie algebra of a jet space. Let $\mathfrak{X}=L_{0} \oplus \cdots \oplus L_{k}$ be the stratified Lie algebra of a jet space $J^{k}\left(\mathbb{R}^{m}, \mathbb{R}^{n}\right)$. From (13) it follows that the center of $\mathfrak{X}$ coincides with $L_{k}$. Let $\mathfrak{I}$ be a stratified ideal of $\mathfrak{X}$. Again, let us assume that there exists a strata preserving Lie algebra isomorphism

$$
\iota: \mathfrak{g} \rightarrow \mathfrak{X} / \mathfrak{I}
$$

Clearly, $\mathfrak{X} / \mathfrak{I}$ must be of step two. The bracket relations (13) have a useful consequence.

Proposition 1 If $\mathfrak{I}$ contains $X_{j}$, for some $j=1, \ldots, m$, then $\mathfrak{I} \supset L_{1} \oplus \cdots \oplus L_{k}$.

Proof Using (13), we can easily see that each $X_{j}$ generates the whole space $L_{1} \oplus \cdots \oplus L_{k}$.

Let us consider the canonical projection $\pi: \mathfrak{X} \rightarrow \mathfrak{X} / \mathfrak{I}$. Proposition 1 implies that $\mathfrak{X} / \mathfrak{I}$ has step two only if $\pi\left(X_{j}\right) \neq 0$, for every $j=1, \ldots, m$ or, in other words, if none of $X_{j}$ 's belongs to $\Im$. Let us now consider the isomorphism $\iota$. The dimensions of the horizontal space and of the center must be preserved. Since $\operatorname{dim} \mathfrak{h}=4$ and $\pi\left(X_{j}\right) \neq 0$ for all $j$ 's, then $m \leq 4$. We prove that $\iota$ is not a Lie algebra isomorphism studying the cases as $m$ varies from 4 to 1 .

(i) $m=4$. In this case the set of vectors $\left\{\pi\left(X_{j}\right): j=1, \ldots, 4\right\}$ generates the first level of $\mathfrak{X} / \mathfrak{I}$. Then $\pi\left(\frac{\partial}{\partial u_{\bar{I}}^{l}}\right)=0$ for every $I \in I(k)$. By (13), we conclude that $L_{1} \subset \mathfrak{I}$, so that $\mathfrak{X} / \mathfrak{I}$ would be commutative, a contradiction.

(ii) $m=3$. If $\pi\left(\frac{\partial}{\partial u_{I}^{l}}\right)=0$ for every $I \in I(k)$, then

$$
\mathfrak{X} / \mathfrak{I}=\operatorname{span}\left\{\pi\left(X_{1}\right), \pi\left(X_{2}\right), \pi\left(X_{3}\right)\right\},
$$

which is not the case. Therefore, a basis of the first stratum of $\mathfrak{X} / \mathfrak{I}$ is given by

$$
\mathcal{B}=\left\{\pi\left(X_{1}\right), \pi\left(X_{2}\right), \pi\left(X_{3}\right), \pi\left(\frac{\partial}{\partial u_{I}^{l}}\right)\right\},
$$

for some $I \in I(k)$ and $l \in\{1, \ldots, n\}$. Since $\pi\left(X_{1}\right), \pi\left(X_{2}\right)$ and $\pi\left(X_{3}\right)$ commute and the first stratum of $\mathfrak{X} / \mathfrak{I}$ cannot contain central elements (since $\mathfrak{h}$ does not), we conclude that the rank 
of $\operatorname{ad} \pi\left(\frac{\partial}{\partial u_{I}^{l}}\right)$ is equal to the dimension of $\mathfrak{z}$, that is three. Therefore, the mapping

$$
\begin{aligned}
\pi\left(X_{1}\right) & \rightarrow e_{1}^{1} \\
\pi\left(X_{2}\right) & \rightarrow e_{1}^{2} \\
\pi\left(X_{3}\right) & \rightarrow e_{1}^{3} \\
\pi\left(\frac{\partial}{\partial u_{I}^{l}}\right) & \rightarrow e_{1},
\end{aligned}
$$

defines a Lie algebra isomorphism between $\mathfrak{X} / \mathfrak{I}$ and $J^{1}\left(\mathbb{R}, \mathbb{R}^{3}\right)$, which is not isomorphic to $\mathfrak{g}$, as we proved in Sect. 3.1.

(iii) $m=2$. Here, $\pi\left(X_{1}\right)$ and $\pi\left(X_{2}\right)$ are nonzero and independent vectors lying in the first level of $\mathfrak{X} / \mathfrak{I}$. Since $m=2, I=\left(i_{1}, i_{2}\right)$. If $\pi\left(\frac{\partial}{\partial u_{I}^{l}}\right)=0$ for every $I \in I(k)$, then $\mathfrak{X} / \mathfrak{I}=\operatorname{span}\left\{\pi\left(X_{1}\right), \pi\left(X_{2}\right)\right\}$, that can not be the case. Hence there exists $I=(i, k-i)$ such that $\pi\left(\frac{\partial}{\partial u_{I}^{l}}\right) \neq 0$ for which

$$
\left[\pi\left(X_{1}\right), \pi\left(\frac{\partial}{\partial u_{(i, k-i)}^{l}}\right)\right]= \begin{cases}\pi\left(\frac{\partial}{\partial u_{(i-1, k-i)}^{l}}\right) & \text { if } i \geq 1 \\ 0 & \text { if } i=0\end{cases}
$$

and

$$
\left[\pi\left(X_{2}\right), \pi\left(\frac{\partial}{\partial u_{(i, k-i)}^{l}}\right)\right]= \begin{cases}\pi\left(\frac{\partial}{\partial u_{(i, k-i-1)}^{l}}\right) & \text { if } i<1 \\ 0 & \text { if } i=k\end{cases}
$$

Since $i$ cannot be equal to 0 and $k$ at the same time, one of the brackets above must be different from zero. Assume that the bracket in (16) and therefore $\pi\left(\frac{\partial}{\partial u_{(i-1, k-i)}^{l}}\right)$ is not zero. On the other hand one has

$$
\left[\pi\left(X_{2}\right), \pi\left(\frac{\partial}{\partial u_{(i-1, k-i+1)}^{l}}\right)\right]=\pi\left(\frac{\partial}{\partial u_{(i-1, k-i)}^{l}}\right),
$$

which implies that $\pi\left(\frac{\partial}{\partial u_{(i-1, k-i+1)}^{l}}\right) \neq 0$. Therefore

$$
\mathcal{B}=\left\{\pi\left(X_{1}\right), \pi\left(X_{2}\right), \pi\left(\frac{\partial}{\partial u_{(i, k-i)}^{l}}\right), \pi\left(\frac{\partial}{\partial u_{(i-1, k-i+1)}^{l}}\right)\right\}
$$

is a basis of the first stratum of $\mathfrak{X} / \mathfrak{I}$. The remaining possibly nonzero brackets between vectors in $\mathcal{B}$ are

$$
\left[\pi\left(X_{1}\right), \pi\left(\frac{\partial}{\partial u_{(i-1, k-i+1)}^{l}}\right)\right]=\pi\left(\frac{\partial}{\partial u_{(i-2, k-i+1)}^{l}}\right)
$$

and

$$
\left[\pi\left(X_{2}\right), \pi\left(\frac{\partial}{\partial u_{(i, k-i)}^{l}}\right)\right]=\pi\left(\frac{\partial}{\partial u_{(i, k-i-1)}^{l}}\right) .
$$

Since $\operatorname{dim}_{\mathfrak{z}}=3, \iota$ is an isomorphism if and only if $\pi\left(\frac{\partial}{\partial u_{(i-1, k-i)}^{l}}\right), \pi\left(\frac{\partial}{\partial u_{(i-2, k-i+1)}^{l}}\right)$ and $\pi\left(\frac{\partial}{\partial u_{(i, k-i-1)}^{l}}\right)$ are different from zero and linear independent. Now, consider the element 
$H_{1} \in \mathfrak{h}$ and the equations

$$
\iota\left[H_{1}, \mathfrak{h}\right]=\left[\iota\left(H_{1}\right), \iota(\mathfrak{h})\right]=\iota\left(\mathbb{R} Z_{1}\right) .
$$

Since ad $H_{1}$ has rank one, also ad $\iota\left(H_{1}\right)$ does. We show that $\mathfrak{X} / \mathfrak{I}$ does not have vectors whose adjoint representation has rank one. A vector in the first stratum of $\mathfrak{X} / \mathfrak{I}$ is of the form $v=a_{1} \pi\left(X_{1}\right)+a_{2} \pi\left(X_{2}\right)+b_{1} \pi\left(\frac{\partial}{\partial u_{(i, k-i)}^{l}}\right)+b_{2} \pi\left(\frac{\partial}{\partial u_{(i-1, k-i+1)}^{l}}\right)$, for some $a_{1}, a_{2}, b_{1}, b_{2} \in \mathbb{R}$. Then ad $v$ has rank one if and only if

$$
\begin{aligned}
{\left[v, \pi\left(X_{1}\right)\right] } & =c_{1} w, \quad\left[v, \pi\left(X_{2}\right)\right]=c_{2} w, \\
{\left[v, \pi\left(\frac{\partial}{\partial u_{(i, k-i)}^{l}}\right)\right] } & =c_{3} w, \quad\left[v, \pi\left(\frac{\partial}{\partial u_{(i-1, k-i+1)}^{l}}\right)\right]=c_{4} w,
\end{aligned}
$$

with $w \in \operatorname{span}\left\{\pi\left(\frac{\partial}{\partial u_{(i-1, k-i)}^{l}}\right), \pi\left(\frac{\partial}{\partial u_{(i-2, k-i+1)}^{l}}\right), \pi\left(\frac{\partial}{\partial u_{(i, k-i-1)}^{l}}\right)\right\}$ and $c_{1}, c_{2}, c_{3}, c_{4} \in \mathbb{R}$. Using (16), (17), (18) and (19), a long but elementary calculation shows that the system (20) does not have any solution for $v \neq 0$. This shows that $\iota$ is not an isomorphism of Lie algebras.

(iv) $m=1$. Here $\pi\left(X_{1}\right) \neq 0$ and the image of $\operatorname{ad}\left(\pi\left(X_{1}\right)\right)$ has necessarily dimension three. Since $m=1, L_{0}=\operatorname{span}\left\{X_{1}\right\} \oplus \operatorname{span}\left\{\frac{\partial}{\partial u_{k}^{l}}: l=1, \ldots, n\right\}$. Therefore, a basis of the first stratum of $\mathfrak{X} / \mathfrak{I}$ is given by $\pi\left(X_{1}\right)$ and the projection of three vectors in $\operatorname{span}\left\{\frac{\partial}{\partial u_{k}^{l}}\right.$ : $l=1, \ldots, n\}$, say $\pi\left(v_{1}\right), \pi\left(v_{2}\right)$ and $\pi\left(v_{3}\right)$. Since $\operatorname{span}\left\{\pi\left(v_{1}\right), \pi\left(v_{2}\right), \pi\left(v_{3}\right)\right\}$ is an abelian subalgebra, the Lie algebra $\mathfrak{X} / \mathfrak{I}$ is isomorphic to the Lie algebra of $J^{1}\left(\mathbb{R}, \mathbb{R}^{3}\right)$ that we considered in Sect. 3.1 and the same argument used there implies that $\mathfrak{g}$ is not isomorphic to $\mathfrak{X} / \mathfrak{I}$.

\subsection{Hessenberg manifolds}

The Lie algebra $\mathfrak{g}$ of the example has the following interpretation. Consider $\mathfrak{s l}(5, \mathbb{R})$ and its Iwasawa decomposition $\mathfrak{k} \oplus \mathfrak{a} \oplus \mathfrak{n}$. Then write the decomposition of $\mathfrak{n}$ according to the root space decomposition of $\mathfrak{s l}(5, \mathbb{R})$ :

$$
\mathfrak{n}=\mathfrak{g}_{\alpha} \oplus \mathfrak{g}_{\beta} \oplus \mathfrak{g}_{\gamma} \oplus \mathfrak{g}_{\delta} \oplus \mathfrak{g}_{\alpha+\beta} \oplus \mathfrak{g}_{\beta+\gamma} \oplus \mathfrak{g}_{\gamma+\delta} \oplus \mathfrak{g}_{\alpha+\beta+\gamma} \oplus \mathfrak{g}_{\beta+\gamma+\delta} \oplus \mathfrak{g}_{\alpha+\beta+\gamma+\delta}
$$

where $\alpha, \beta, \gamma$ and $\delta$ are the simple roots. The subspace $\mathfrak{n}_{\mathcal{C}}=\mathfrak{g}_{\alpha+\beta+\gamma} \oplus \mathfrak{g}_{\beta+\gamma+\delta} \oplus \mathfrak{g}_{\alpha+\beta+\gamma+\delta}$ is an ideal of $\mathfrak{n}$. Therefore $\mathfrak{n} / \mathfrak{n}_{\mathcal{C}}$ is a Lie algebra. If $p: \mathfrak{n} \rightarrow \mathfrak{n} / \mathfrak{n}_{\mathcal{C}}$ is the natural projection, then $p\left(\mathfrak{g}_{\alpha+\beta}\right)=p\left(\left[\mathfrak{g}_{\alpha}, \mathfrak{g}_{\beta}\right]\right)=\left[p\left(\mathfrak{g}_{\alpha}\right), p\left(\mathfrak{g}_{\beta}\right)\right]$. From this and similar observations, it follows that $\mathfrak{n} / \mathfrak{n}_{\mathcal{C}}$ is a stratified nilpotent Lie algebra. The Lie algebra $\mathfrak{n} / \mathfrak{n}_{\mathcal{C}}$ is isomorphic as a vector space to the sum

$$
p\left(\mathfrak{g}_{\alpha}\right) \oplus p\left(\mathfrak{g}_{\beta}\right) \oplus p\left(\mathfrak{g}_{\gamma}\right) \oplus p\left(\mathfrak{g}_{\delta}\right) \oplus p\left(\mathfrak{g}_{\alpha+\beta}\right) \oplus p\left(\mathfrak{g}_{\beta+\gamma}\right) \oplus p\left(\mathfrak{g}_{\gamma+\delta}\right) .
$$

Then the vectors $H_{1}, K_{1}, K_{2}, H_{2}, Z_{1}, Z_{2}, Z_{3}$ of $\mathfrak{g}$ can be identified with a basis of $\mathfrak{n} / \mathfrak{n}_{\mathcal{C}}$ with respect to the above decomposition. In [4], we study the problem of contact mappings for Hessenberg manifolds. These are smooth submanifolds of homogeneous spaces S/P, where $\mathrm{S}$ is a semisimple Lie group and $\mathrm{P}$ is the minimal parabolic subgroup of $\mathrm{S}$ [3]. Hessenberg manifolds are defined by the choice of a subset $\mathcal{R}$ of the set of positive roots $\Sigma_{+}$which are behind the structure of the semisimple group $\mathrm{S}$. If $\operatorname{Lie} S=\mathfrak{s t}(5, \mathbb{R})$ and $\mathcal{R}=\{\alpha, \beta, \gamma, \alpha+$ $\beta, \beta+\gamma, \gamma+\delta\}$, then the tangent space of the Hessenberg manifold corresponding to these data is identified with $\mathfrak{n} / \mathfrak{n}_{\mathcal{C}}$. In fact, we can use Theorem 1 in order to construct a class of Hessenberg manifolds that are nonrigid. 


\section{References}

1. Corwin, L., Greenleaf, F.P.: Representations of Nilpotent Lie Groups and Their Applications. Cambridge University Press, Cambridge (1990)

2. Cowling, M., De Mari, F., Korányi, A., Reimann, H.M.: Contact and conformal mappings in parabolic geometry. I. Geom. Dedicata 111, 65-86 (2005)

3. De Mari, F., Pedroni, M.: Toda flows and real Hessenberg manifolds. J. Geom. Anal. 9(4), 607-625 (1999)

4. Ottazzi, A.: Multicontact vector fields on Hessenberg manifolds. J. Lie Theory 15, 357-377 (2005)

5. Reimann, H.M.: Rigidity of H-type groups. Math. Z. 237(4), 697-725 (2001)

6. Reimann, H.M., Ricci, F.: The complexified Heisenberg group. In: Proceedings on Analysis and Geometry (Russian) Novosibirsk Akademgorodok, pp. 465-480 (1999)

7. Rigot, S.: Counter example to the Besicovitch covering property for some Carnot groups equipped with their Carnot-Carathéodory metric. Math. Z. 248, 827-848 (2004)

8. Saunders, D.J.: The Geometry of Jet Bundles, vol. 142. London Mathematical Society Lecture Note Series. Cambridge University Press, Cambridge (1989)

9. Warhurst, B.: Jet spaces as nonrigid Carnot groups. J. Lie Theory 15, 341-356 (2005)

10. Yamaguchi, K.: Differential systems associated with simple graded Lie algebras. In: Progress in differential geometry. Adv. Stud. Pure Math. 22, Math. Soc. Japan, Tokyo 1993, pp. 413-494 